\title{
An Experimental Study on the Role of Touch in Shared Virtual Environments
}

\author{
CAGATAY BASDOGAN, CHIH-HAO HO, MANDAYAM A. SRINIVASAN \\ Laboratory for Human and Machine Haptics \\ Massachusetts Institute of Technology, U.S.A \\ MEL SLATER \\ University College London, U.K.
}

\begin{abstract}
Investigating virtual environments has become an increasingly interesting research topic for engineers, computer and cognitive scientists, and psychologists. Although there have been several recent studies focused on the development of multimodal virtual environments (VEs) to study human-machine interactions, less attention has been paid to human-human and human-machine interactions in shared virtual environments (SVEs), and to our knowledge, no attention paid at all to what extent the addition of haptic communication between people would contribute to the shared experience. We have developed a multimodal shared virtual environment and performed a set of experiments with human subjects to study the role of haptic feedback in collaborative tasks and whether haptic communication through force feedback can facilitate a sense of being and collaborating with a remote partner. The study concerns a scenario where two participants at remote sites must co-operate to perform a joint task in a SVE. The goals of the study are (1) to assess the impact of force feedback on task performance, (2) to better understand the role of haptic communication in human-human interactions, (3) to study the impact of touch on the subjective sense of collaborating with a human as reported by the participants based on what they could see and feel, and (4) to investigate if gender, personality, or emotional experiences of users can affect haptic communication in SVEs. The outcomes of this research can have a powerful impact on the development of next generation human-computer interfaces and network protocols that integrate touch and force feedback technology into the Internet, development of protocols and techniques for collaborative teleoperation such as hazardous material removal, space station repair, and remote surgery, and enhancement of virtual environments for performing collaborative tasks in shared virtual worlds on a daily basis such as co-operative teaching, training, planning and design, cybergames, and social gatherings. Our results suggest that haptic feedback significantly improves the task performance and contributes to the feeling of 'sense of togetherness' in SVEs. In addition, the results show that the experience of visual feedback only at first, and then subsequently visual plus haptic feedback elicits a better performance than presentation of visual plus haptic feedback first followed by visual feedback only.
\end{abstract}

Categories and Subject Descriptors: I.3.6 [Computer Graphics]: Methodology and Techniques-interaction techniques, ThreeDimensional Graphics and Realism-virtual reality; I.2.9 [Artificial Intelligence]: Robotics-manipulators; H.5.2 [Information Interfaces and Presentation]: User Interfaces-input devices and strategies

General Terms: Design, Experimentation, Human Factors, Measurement, Performance

Additional Key Words and Phrases: shared virtual environments, force-feedback devices, haptic interaction, co-presence.

Authors' addresses: C. Basdogan, Jet Propulsion Laboratory, California Institute of Technology, 4800 Oak Grove Drive, M/S: T1089B, CA, 91109; e-mail: Cagatay.Basdogan@jpl.nasa.gov; C. Ho, M.A. Srinivasan, Laboratory for Human and Machine Haptics, Department of Mechanical Engineering and, Research Laboratory of Electronics, Massachusetts Institute of Technology, Cambridge, MA, 02139; e-mails: chihhao, srini@mit.edu; M. Slater, Department of Computer Science, University College London, Gower Street, London, WC1E 6BT, U.K.; e-mail: m.slater@ cs.ucl.as.uk 


\section{Introduction}

Virtual reality involves the creation of multi-sensory experience through artificial means. The effectiveness of virtual environments has often been linked to the richness of sensory information and realness of the experience (Held and Durlach, 1992; Ellis, 1992; Barfield and Furness, 1995). The quantification of the participant's multisensory experience in these environments involves a set of objective measures that quantify a particular task performance as well as a set of measures that quantify the subjective experience (Sheridan, 1992a).

Although the development of virtual worlds that can provide a multi-sensory experience to a single user is receiving substantial attention from researchers, less attention has been given to shared virtual environments (SVEs) and shared experience. In general, single user VR applications involve the visualization of a scene and interaction with objects within the scene. However, the fundamental aspect of shared experience is the sensory communication that takes place between participants which enables them to display their actions and express their emotions to each other. To explore possible uses of SVEs for learning, entertainment and work, Waters, Barrus (1997) and their colleagues have developed 'Diamond Park': a virtual landscape park that accommodates both bicycling and social interactions. Macedonia and Noll (1997) discuss the possibilities of constructing a SVE for conducting scientific experiments globally with remotely located participants. The applications involve the training of astronauts and telediagnosis. Jasnoch et al. (1996) describe a SVE for virtual prototyping and emphasize that such an environment can decrease product development time and cost and increase the quality and flexibility in computer-aided design. Benford and colleagues have carried out a series of studies on collaborative virtual environments, most recently (Benford et. al., 1998) exploring boundaries between the real and the virtual in the context of a poetry performance. As part of the European Collaborative Virtual Environments (COVEN) project (Normand et al., 1999) a series of experimental studies of small group collaboration have been carried out focussing on such issues as emergence of leaders, ability to interact, and role of the avatars (Tromp et al., 1998; Steed, et al., 1999; Slater et al., 2000).

All of the studies above made use of visual and auditory modalities for communication and interaction. Schroder (1997) claims that investigating the sensory and social interactions between networked VR users can lead to the development of new VR systems that are more adaptive to the needs of users. These interactions will increase as more sensory modalities that are used on an everyday basis are integrated into the networked VR systems and personalized to the needs of the user. For example, several studies have already shown that the visual communication that takes place via gesture, facial expression, and body posture together with auditory communication through text display or voice are important and produce a positive effect on the participant's sensory experience in virtual environments (Welch et al., 1996; Barfield and Furness, 1994 and Stanney et al., 1998a). Stanney et al. (1998b) discuss several factors, including task and user characteristics, multimodal interaction, human sensory and motor physiology, cybersickness, psychological aftereffects, and social impact of technology, in analyzing human factors issues in virtual environments.

One of the sensory modalities that need a more detailed investigation in this regard is the 'haptic channel'. Being able to explore and manipulate objects through haptic interfaces in virtual environments has several applications in various fields including medicine, education, entertainment, and CAD (Srinivasan and Basdogan, 1997; Salisbury and Srinivasan, 1997). The research in the area of haptic interfaces and rendering techniques has grown significantly during the last few years and initial results have already shown the significant role of haptics in the sensory experience of user. Moreover, in the area of teleoperators, studies have shown that operator performance increases significantly in telemanipulation of remote objects when haptic feedback is provided (Das et al., 1992). Sheridan and colleagues (1992b) have conducted experiments on automation and planning of complex tasks through human supervisory control and have shown that the task completion times can be reduced with haptic feedback. It also appears that touching and manipulating objects improves the subjective experience. Durlach and Slater (2000) emphasize that touch, in comparison to other sensory modalities, is more local and bi-directional which is linked to closeness and intimacy. Our research in this area also supports these studies and demonstrates the positive impact of haptic on task performance and subjective sense of togetherness in SVEs (Ho et al., 1998; Basdogan et al., 1998).

Although it seems natural to think that the addition of touch will improve task performance and increase the subjective sense of 'being' and 'collaborating' with someone in SVEs, to our knowledge, the impact of touch and force-feedback has not been investigated in detail. The particular research questions that arise are: 
?? How can we investigate the effect of touch on the task performance in SVEs? For example, what types of measures can be defined to quantify the performance and subjective experience?

?? Which aspects of touch are the most important for haptic communication in shared environments? For example, how does force magnitude and direction effect the multi-sensory experience of participants?

?? What types of analytical models and paradigms (e.g. fuzzy logic, statistical learning models, supervisory control, etc.) can be developed to explain the haptic interactions between participants in SVEs? In what ways will the subjective experience of participants in SVEs depend on the haptic interaction models and the haptic stimuli employed? For example, what types of force control strategies do people follow to manipulate objects and interact with others to emphasize their 'presence' in shared environments?

?? Can haptic communication enable us to assess personality characteristics of the remote partner? If so, how does knowing this kind of information affect the performance of partners and their subjective experience? Can we improve the performance of our remote partner if we feel and sense his/her character through touch? Or, if a remote partner guesses your gender and some characteristics of your personality through touch feedback during a collaborative task, will this make you comfortable/uncomfortable and will this effect your performance and sense of togetherness?

?? How are the answers to these questions influenced by time delays and the events taking place in the visual channel? For example, will the delays in force or visual display make the subjects believe that they are more likely to be interacting with an artificial entity (e.g. a computer) instead of a real person? Or, if we feel different from what we expect to feel based on what we see in the visual scene, how will this effect our task performance and our sense of 'being with someone'?

Recent hardware and software advances in haptic interfaces and personal computers enable us to integrate force feedback into SVEs and study these interesting questions. With the current technology and our experience in multimodal virtual environments involving haptic interfaces, we can render visual and haptic images in real-time and integrate multiple sensory modalities into a low-cost virtual reality system for investigating the nature of haptic interactions between two people in a SVE. In addition to studying the haptic interactions between humans, our future studies will explore the haptic interactions between a human being and a computer.

Our objective in this research is to identify the necessary components of touch that is required to make a human participant believe that he/she interacts with another human being. To achieve this goal, we will first need to identify the important components of haptic communication that takes place between human beings in SVEs. Specifically, the proposed research provides a necessary foundation for the incorporation of 'personal' touch and force feedback devices into networked VR systems and the Internet by investigating the haptic interactions that take place between humans in SVEs. Ultimately a goal could be to create a haptic avatar which behaves in such a way that it cannot be distinguished from a human participant. These ideas are summarized in Figure 1. 


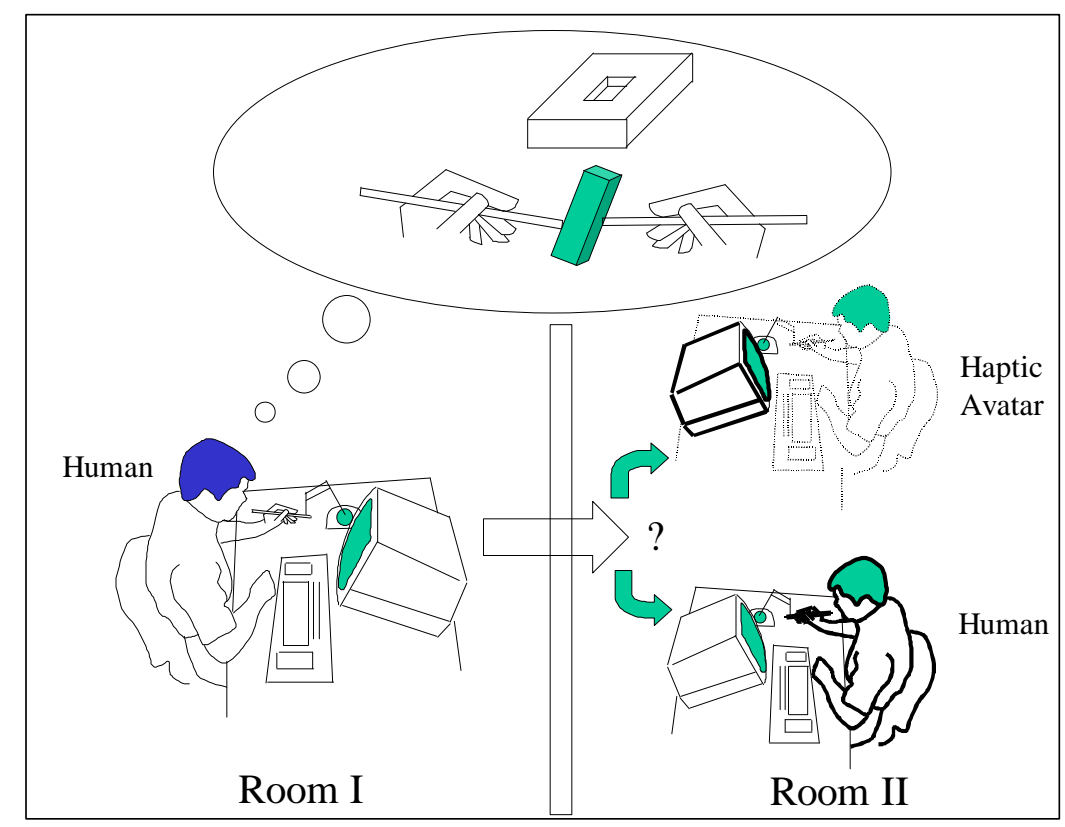

Figure 1. Our future experiments will involve the haptic version of the 'Turing' test: Haptic devices can be programmed to imitate the haptic interactions that take place between human beings. The experiments that we have proposed for human interactions in this study can be repeated with the condition that the remote human partner switches back and forth, in a random order, with a computer. For each trial, the subject can be asked to identify whether he/she interacts with a human being or a computer.

The goal of our current study is to investigate the influence of haptic feedback on the task performance of participants and also their sense of togetherness. The outcomes of this research can have a powerful impact on the development of next generation human-computer interfaces and network protocols that integrate touch and force feedback technology into the Internet, development of protocols and techniques for collaborative teleoperation such as hazardous material removal, space station repair, and remote surgery, and enhancement of virtual environments for performing collaborative tasks in shared virtual worlds on a daily basis such as co-operative teaching, training, planning and design, cybergames, and social gatherings.

In the next section, we first discuss the development of a multi-user VR set-up that enables two human subjects at remote locations to interact with each other in SVEs. Design of the experiment is discussed in section 3. In section 4, we describe the haptic interaction model that enables the haptic communication between participants. In section 5, we define a set of performance and subjective measures to analyze the results of the experiments. The results and conclusions of the study are summarized in sections 6 and 7, respectively.

\section{Development of a multi-user VR set-up for experiments}

Previously, we have developed a single-user VR system that integrates visual and haptic displays and enables a user to interact with virtual objects. The hardware components of the set-up include an IBM compatible PC (Dual Pentium II $300 \mathrm{MHz}$ processors) with a high-end 3D graphics accelerator for visualization of virtual objects and a PHANToM (SensAble Technologies Inc.) to simulate haptic sensations. This system has been successfully tested in our previous studies on haptic rendering of 3D objects and their surface properties such as texture and friction (Basdogan et. al, 1997; Ho et. al., 1999). During the simulations, the user manipulates the generic stylus of the force-feedback device and feels the reaction forces that arise from interactions with the computer generated 3D objects. The software modules of this system that enable haptic interactions in virtual environments can be summarized as follows: 
(1) Multi-Threading Techniques for Integration of Vision and Touch: To have a satisfying experience in interacting with a VE, the graphics and haptic update rates should be maintained at least around $30 \mathrm{~Hz}$ and $1000 \mathrm{~Hz}$, respectively. We have developed multi-threading techniques and successfully synchronized the visual and haptic servo loops (Ho et al. 1999). In our model, the haptics and graphics processes are server and client, respectively.

(2) Haptic Rendering Techniques: We have developed efficient haptic rendering techniques for displaying the feel of 3D polyhedral objects in VEs (Basdogan et. al, 1997; Ho et. al., 1999). Using the proposed haptic rendering method with a force reflecting haptic interface device, users can sense and feel the shape and surface details of virtual objects. The major components of our rendering method are a set of rule-based algorithms and a hierarchical search tree for detecting collisions between the generic probe (end-effector) of a force-reflecting haptic device and polygonal objects in VEs. Smooth rigid, texture mapped, dynamic, and deformable objects have been successfully simulated in virtual environments using our rendering technique (Ho et al., 1999).

(3) User/Developers Interface: A simple, but expandable user interface was developed using Open Inventor graphics tool kit and $\mathrm{C}++$ programming language to display 3D objects in virtual environments. The interface enables the user to load polyhedral virtual objects from a simple user-defined text file for constructing a multi-modal VE that has visual and haptic components. After loading the Inventor object files (i.e. 3D objects that are made of triangles), the coordinates of the vertices and the indices of the polygons are automatically stored in our hierarchical database for detecting collisions between virtual objects in the scene and the haptic stylus. This interface is flexible enough to be extended by the end-user. Using this text file, the user can intuitively add (or subtract) 3D polyhedral objects into (from) the virtual scene and assign visual and haptic properties to the objects.

We have extended our existing single-user VR system to accommodate two people who are located at remote sites, share the same virtual environment, and interact with each other via their personal haptic interfaces. To eliminate uncontrolled time delays that may occur when two computers communicate over a network, we use a single computer (with dual $300 \mathrm{MHz}$ processors) and synchronize two monitors and two PHANToM force feedback devices (one for each subject). We have done this so that we can study the nature of the haptic interactions between people through virtual touch, under controlled conditions-without the time delays induced by a network. Such a setup (one computer running two haptic devices) has been tested in our laboratory and shown to provide real-time and stable visual and haptic interactions. We made cable and constructional arrangements to move one PHANToM and one monitor to another room and to connect to the master computer (The video signal generated by the master computer is bifurcated to display the same image in two monitors located in two separate rooms).

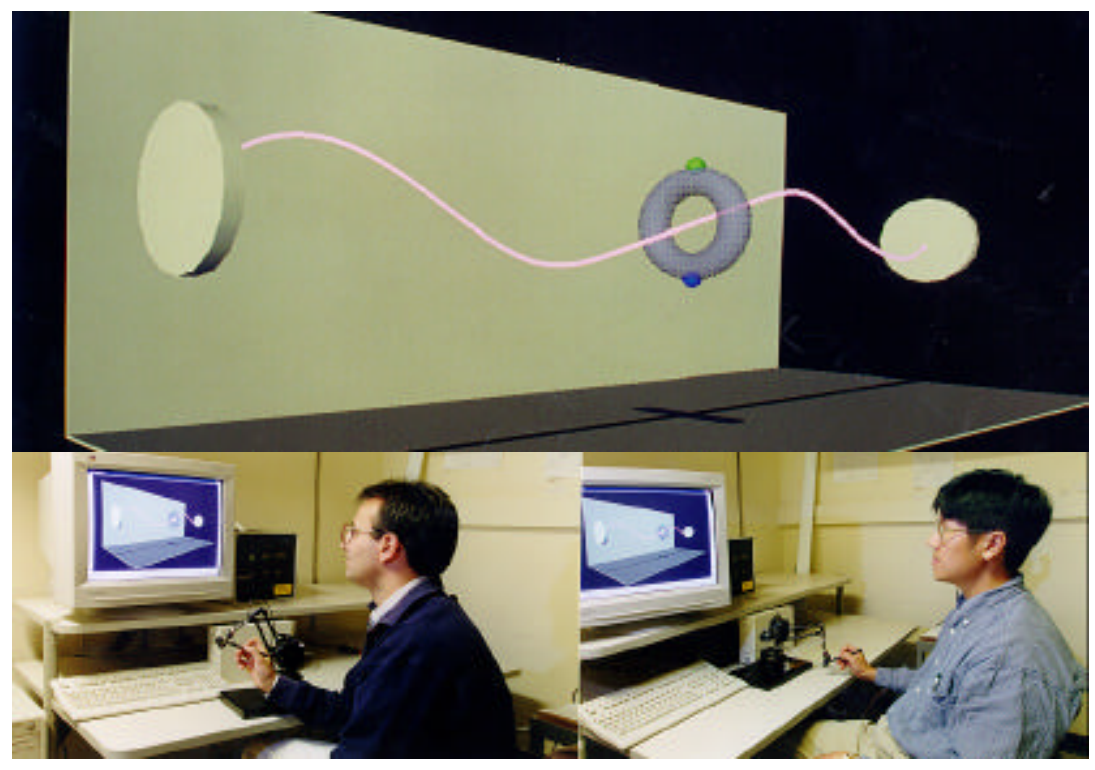

Figure 2. Our current VR set-up that enables two people, at remote locations, to interact with each other through visual and haptic displays. For example, subjects will be able to hold and move a ring on a wire in a collaborative manner as depicted in this figure. To eliminate time delays due to network connections, we bifurcated the signals from a single host and displayed on two separate monitors. To maintain stable force interactions, the haptic update rate was fixed at $1 \mathrm{kHz}$. 


\section{The Experiment}

\subsection{Experimental Design}

During the experiment, subjects were asked to perform a collaborative task in a SVE. The subjects were not allowed to meet and know the remote partner, and did not know where partners were located. The participants were in different rooms (each player entered the room from a different door) but shared a common visual scene and felt the objects in the scene through their personal haptic devices (Figure 2). 10 subjects participated in the experiment. There were two experimental conditions in the study which, depended on the type of sensory feedback provided to each participant. The following sensory conditions were used for the experiment:

?? only visual feedback $(V)$

?? visual and haptic feedback $(V+H)$

There were at least 15 trials for each experimental condition. Subjects were in two groups. The first group received condition 2 (visual and haptic feedback) first, and then about 10 days later repeated the same trials but with visual feedback only (condition 1). The second group, received condition 1 (only visual feedback) first, and then participated in the visual plus haptic feedback session about 10 days later. All the subjects collaborated with the same remote partner during the experiments. This person was an 'expert' user of the system, and performed, as far as possible, with constant performance throughout (The subjects were not told that their partner was an expert). Thus all the data gathered during the experiment related to the subject only, and not the partner. For each trial, the performance of the subject was recorded to generate a score and displayed to the subject after the trial. Although it was not emphasized during the verbal description of the task to each subject before the experimentation, most of the subjects quickly grasped the importance of time in the computation of the performance score. In general, subjects learned how to perform the task in time, and the time taken to complete the task reached a steady state for almost all the subjects after they completed approximately 10 trials.

\subsection{Scenario and Task}

The task was to move a ring with the help of another person without touching a wire (see Figure 3). A ring, a wire, and two cursors (the small spheres in Figure 3 represent the contact points of each subject) attached to the ring were displayed. The cursors were attached to the haptic devices through virtual springs in both $\mathrm{V}$ and $\mathrm{V}+\mathrm{H}$ conditions. Subjects were asked to move a ring on a wire, back and forth many times, in collaboration with their partner such that contact between the wire and the ring was minimized or avoided (we name this game as 'Ring on a Wire'). If the ring touched the wire, the colors of the ring and the surrounding walls changed to warn the subject of an error. They changed back to their original colors when the subjects corrected the position of the ring. To hold the ring, both collaborators needed to press on the ring in $\mathrm{Z}$ direction towards each other above a threshold force (see Figure 5). If they did not press on the ring at the same time, the ring did not move. To move the ring along the wire, they each needed to apply an additional lateral force in X and Y directions (see Figure 5). 


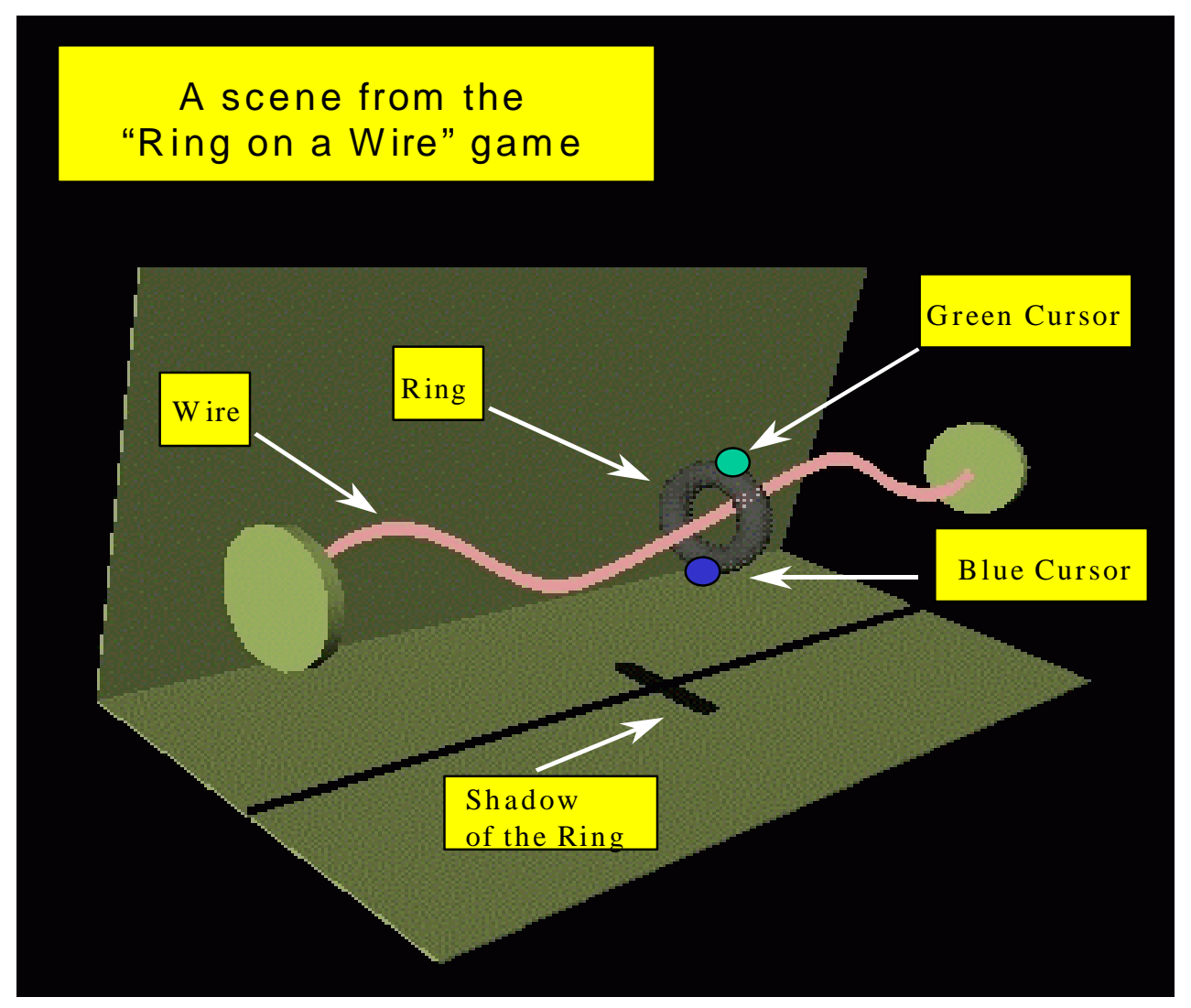

Figure 3. Elements of the 'Ring on a wire' game: The goal of the game is to move the ring on a wire with minimum contact to wire. Each participant has his/her own cursor (blue and green spheres in the picture) to manipulate the ring. Participants could 'feel' each other through their personal force feedback devices. In order to 'hold' the ring, participants need to apply some force towards each other as if they are holding a ring with their index fingers in real life. However, there is no gravity in the experiment and the ring does not fall down if the subjects do not apply enough force to 'hold' it. In order to 'move' the ring, they also need to apply a lateral force simultaneously. The curvature of the wire changes to indicate the next course when participants collaboratively move the ring and reach either ends. Participants can also use the displayed shadows of the ring and the wire to adjust the position of the ring relative to the wire.

\section{Haptic Interaction Models and Paradigms}

For SVEs to be effective in performing collaborative tasks that involve haptic feedback, a model that simulates the haptic interactions among the participants needs to be developed. If network time delays, the difficult nature of some collaborative tasks, and our limited knowledge on sensory integration of vision and touch are all considered, the problem of developing a universal model for haptic interactions could be too complex. Moreover, the participants could follow several different strategies to manipulate the virtual objects during the execution of the task. For example, we could talk about the 'sequential' versus 'simultaneous' types of haptic manipulations (see Figure 4). Or, one could talk about the 'priority-based' versus 'request time dependent' haptic interactions (see Broll, 1995 for a related discussion). Or it is even possible to make one subject 'stronger' than the other. Since our limited knowledge in this area makes it almost impossible to integrate all these features into a single interaction model, the type of model selected to simulate haptic interactions between participants in SVEs depend, at this stage, on the collaborative task itself. 


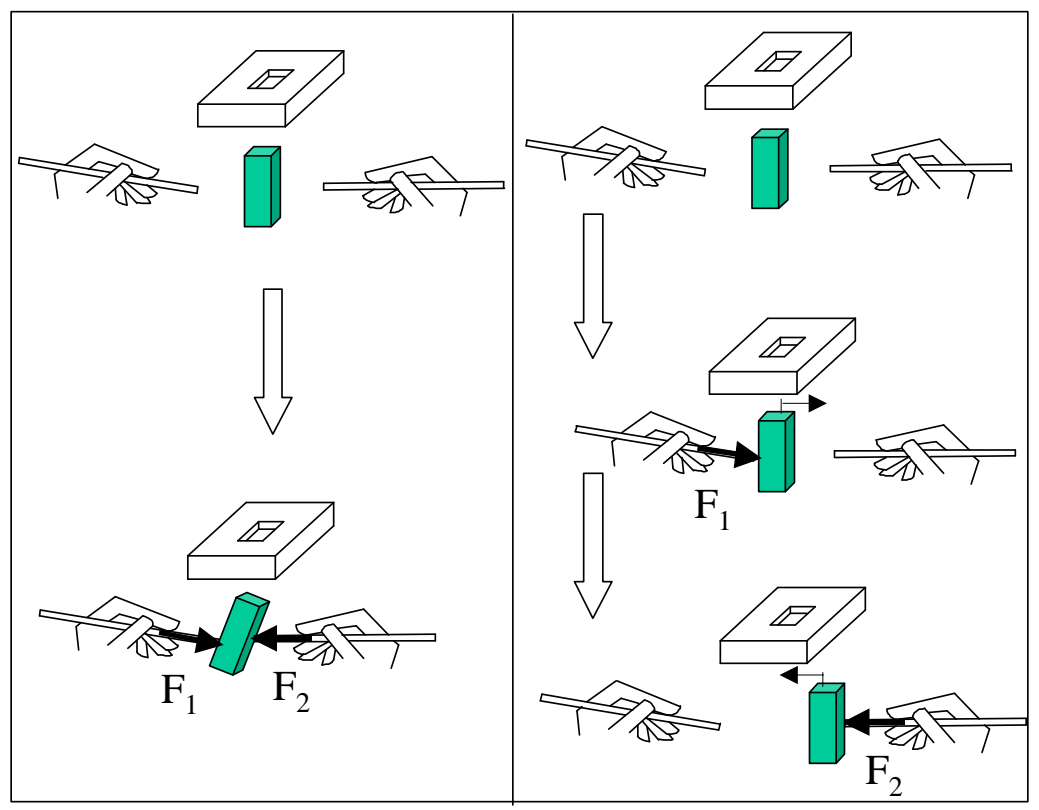

(a)

(b)

Figure 4. The difference between the 'simultaneous' (a) versus 'sequential' (b) haptic manipulation. In the case of 'simultaneous' haptic manipulation, both participants apply a force to hold and manipulate the object. However, in 'sequential' manipulation, participants manipulate the object one at a time.

We have developed a simple physically based model to simulate the haptic interactions between participants. In general, each subject manipulates his/her own cursor through a stylus attached to the force feedback device placed next to his/her seat. When the subject manipulates the stylus of the haptic device with his/her dominant hand, the cursor moves in 3D space, so that the manipulated object translates or rotates depending on the task. In our experiments, a spring-damper model ( $F ? k ? p ? b ? \beta$, where $\mathrm{F}$ is the force exerted by the user to the ring that is felt by his/her remote partner, $\mathrm{k}$ and $\mathrm{b}$ are the spring and the damping coefficients, and $? p$ is the displacement of the subject's cursor) was used to control the impedance of interactions between the participants and between the subject's cursor and the ring in the scene (see Figure 5). This model simply simulates the translational movements of the ring on a wire and pulling and pushing between the subjects. Hence, if a subject pulls or pushes his own cursor, the remote partner feels the forces. Visually, however the ring remained rigid (i.e. no deformation of the ring was displayed graphically). The rotation of the ring due to unbalanced forces applied by the participants was prevented to make the task easier. Moreover only the 'simultaneous' haptic interactions were supported such that the ring did not move if both subjects did not apply sufficient forces to the ring at the same time. Choi et al. (1997) also describe a 'spring-based' model for displaying haptic feedback to multiple remote users connected via network in shared environments. 


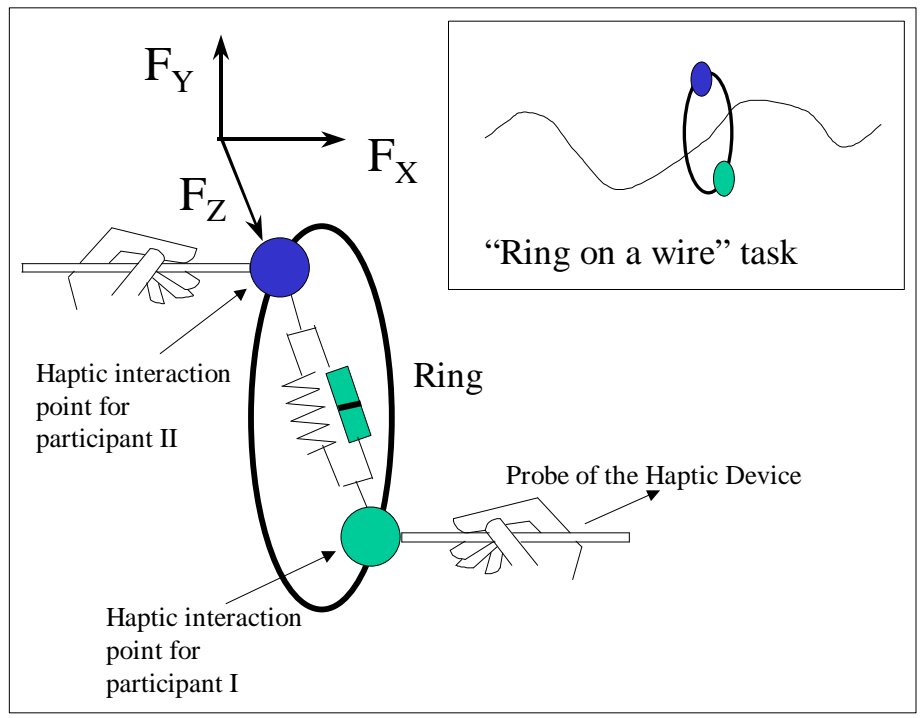

Figure 5. The proposed model for simulating haptic interactions between participants in SVEs to perform a collaborative task of moving a ring on a wire.

\section{Response and Explanatory Variables}

\subsection{Response Variables}

Two main response variables were used, the first to assess success in performance of the task, and the second to assess the subjective assessment of 'togetherness'. We consider each of these in turn.

a) The Performance Measure

A score for the performance of subjects was derived from two types of measurements: (1) total amount of time taken to complete the task (2) the ratio of time spent in 'error-free condition' to total time as:

$$
\text { Score } ? \frac{?\left(R^{2}\right)}{T}
$$

where, ? is a constant, $\mathrm{T}$ is the total time taken to complete the task, and $\mathrm{R}$ is the ratio of error-free time to the total time. Subjects repeated the trials many times, and performance varied considerably within each set of trials. We analyzed the results using the maximum as well as average scores attained across the trials of a particular session. Since there was no significant difference in the results obtained from the two measures, we have shown only the maximum scores in the tables. Other measures have also been suggested in the literature to quantify the performance of the user in tasks that involve force-feedback. For example, Das et al. (1992) suggested the use of cumulative ( ? $f_{i} ? t$, where ? $t$ is the sampling interval) and average $\left(?_{i} f_{i} ? t\right) / N$, where $\mathrm{N}$ is the number of samples) forces exerted by the user, in addition to root-mean-square position error, to quantify the performance for a teleoperated task.

b) The Sense of Being Together

After each session the subjects answered a questionnaire, which supplied the basic demographic and background information and the main variables in which we were interested. We adopted the same strategy as used in other work in shared virtual environments (for example, Slater, 2000). Eight questions on the sense of being together were 
scattered through the questionnaire administered after each session, each question on a 1 to 7 scale. These were as follows:

(1) To what extent, if at all, did you have a sense of being with the other person?

(2) To what extent were there times, if at all, during which the computer interface seemed to vanish, and you were directly working with the other person?

(3) When you think back about your experience, do you remember this as more like just interacting with a computer or working with another person?

(4) To what extent did you forget about the other person, and concentrate only on doing the task as if you were the only one involved?

(5) To what extent were you and the other person in harmony during the course of the performance of the task?

(6) Think about a previous time when you cooperatively worked together with another person in order to move or manipulate some real thing in the world (for example, shifting some boxes, lifting luggage, moving furniture, and so on). To what extent was your experience in working with the other person on this task today like that other real experience, with regard to your sense of doing something together?

(7) During the time of the experience, did you often think to yourself that you were just manipulating some screen images with a pen-like device, or did you have a sense of being with another person?

(8) Overall rate the degree to which you had a sense that there was another human being interacting with you, rather than just a machine?

The overall score was constructed as the number of 'high' ('6' or '7') responses out of the 8 questions. This avoids the problem of averaging ordinal responses, and allows the use of logistic regression.

\subsection{Explanatory Variables}

Several questions were asked in three categories including (1) performance (2) emotional reactions, and (3) personality profile. Each of the questions in categories was rated on a 1-7 scale.

(1) Performance: Each person made a self-assessment of his/her own performance and the performance of the other person using the questionnaire.

(2) Emotional reaction: The response of the subject regarding their own degree of embarrassment during the task, and their assessment of their partner.

(3) Personality profile: Each individual to complete a standard personality profile test (Leary, 1983) supplemented by some additional questions particularly relevant to this task. This test focussed on the degree of social anxiety generally experienced by the person in life, and constructed such that the higher the score the greater the degree of social anxiety.

(4) Assessment of the other person: We also asked subjects to complete the social anxiety test guessing the likely answers for their remote partner. The purpose was to examine whether subjects' assessments of their unknown partner would correlate and change under the experimental conditions.

\section{Results}

\subsection{Performance and the Main Conditions}

Table 1 shows the means and standard deviation of the performance scores for each cell of the experimental design. It is clear that haptic feedback enhances performance considerably, independently of the order of presentation (whether vision or vision plus haptic was the first experience of the subject). The table also suggests a possible nonadditive interaction effect, where the experience of vision first, and then subsequently vision plus haptic generates the highest performance (as suggested by the final cell in the table). In order to test this, an Analysis of Variance model was fitted, including an interaction between the main effects of group and condition. The overall model fit was excellent, with $94 \%$ of the variation in performance explained by the main conditions $\left(R^{2}=0.94\right)$. The interaction effect was highly significant $(\mathrm{t}=5.8$ on 16 d.f.) supporting the idea that order mattered in the following sense: use of the visual system only first, followed by the use of visual and haptic system (group 2) resulted in higher performance than presentation of visual plus haptic first followed by visual only (group 1). In other words, 
the initial visual only experience provided some degree of training, which was significantly enhanced by the addition of haptics.

Table 1. Means and Standard Deviation of Maximum Performance Scores ( $\mathrm{n}=5$ for each cell)

\begin{tabular}{|l|l|l|}
\hline & Group I (V+H first) & Group II (V first) \\
\hline Condition 1 (V) & $67 ? 14$ & $77 ? 25$ \\
\hline Condition 2 (V+H) & $161 ? 39$ & $333 ? 39$ \\
\hline
\end{tabular}

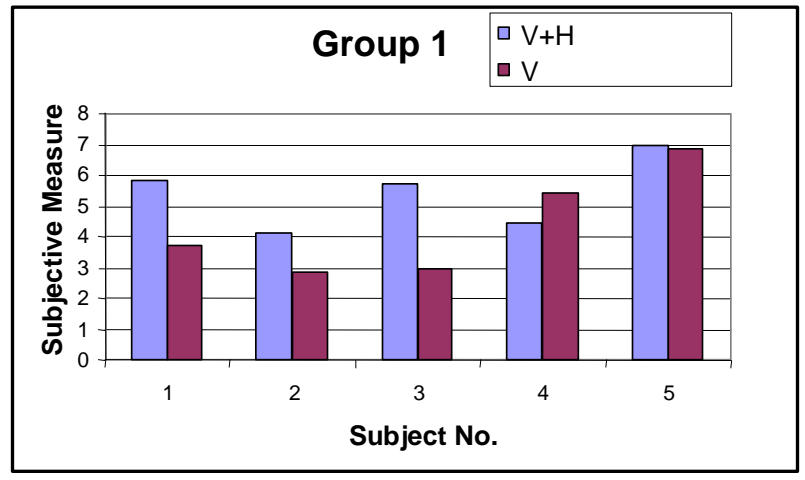

(a)

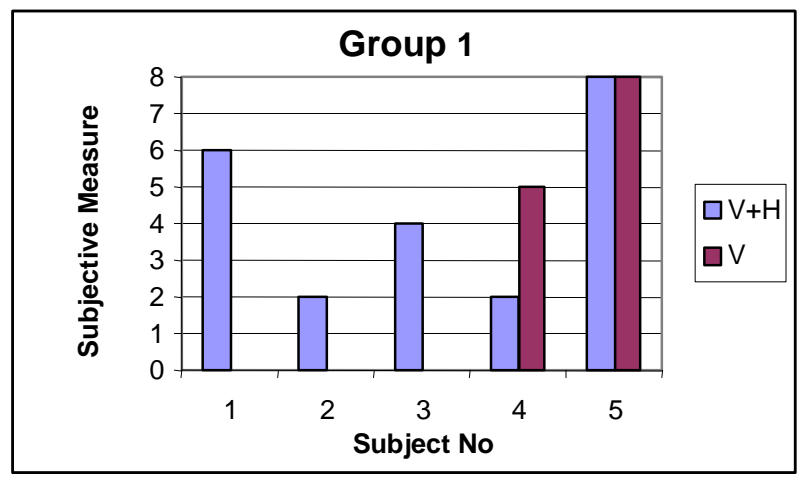

(c)

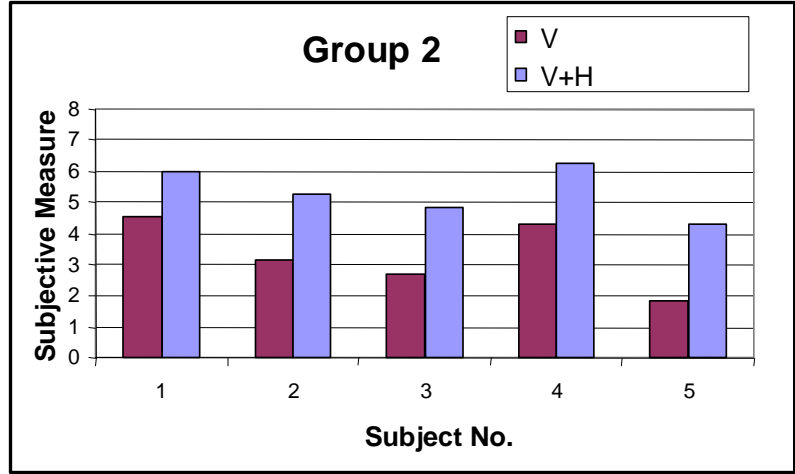

(b)

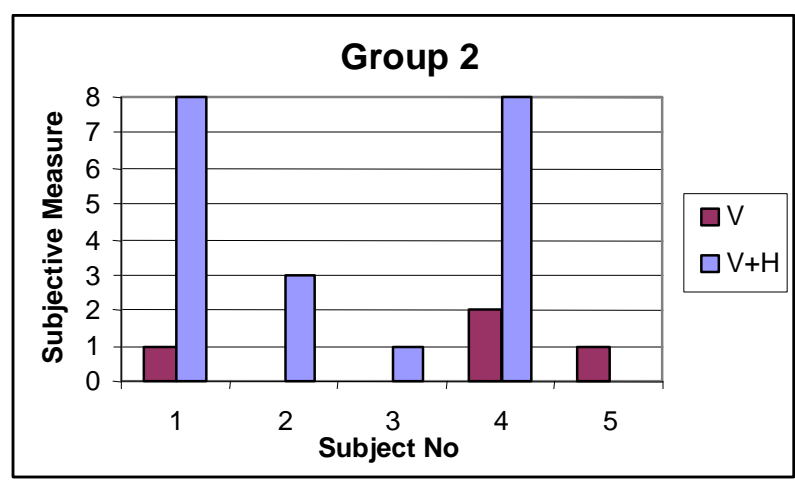

(d)

Figure 6. Subjective scores (i.e. score for "sense of togetherness") of each subject in groups I and II are represented as bar charts for comparison of experimental conditions (condition "V" vs. condition "V+H"). In charts (a) and (b), the subjective measure is based on the average of subjects' responses to the 8 'togetherness' questions. In charts (c) and (d), the subjective measure is based on the number of 'high' (' 6 ' or '7') responses out of the 8 'togetherness' questions. Results show that subjective sense of togetherness increases with the addition of haptic feedback.

\subsection{Togetherness}

Figure 6 suggests that togetherness increased for almost each subject under the haptic condition. In order to further explore the relationship between togetherness and other variables, a multiple logistic regression was carried out (see Table 2). This considered the impact of the main conditions on togetherness, gender, age, and degree of computer usage (only significant results are reported here). Independently of the order of presentation (which was not significant) togetherness was significantly higher with the use of haptic feedback. Togetherness significantly decreased with age, and increased with the degree of computer use. Gender was significant - females reported a higher level of togetherness than males. There was some evidence that the impact of social anxiety was different between males and females. For males, higher anxiety was associated with a lower sense of togetherness whereas for 
females higher social anxiety was associated with a higher sense of togetherness. However, this effect was only significant if age were excluded from the regression model.

Table 2. Logistic regression for "togetherness": measured as a count of high scores out of 8 questions.

\begin{tabular}{|l|l|l|}
\hline Variable & Association with togetherness & $\begin{array}{l}\text { Change in Chi-squared value } \\
\text { for deletion from the logistic } \\
\text { regression model on 1 d.f. } \\
\text { (Chi-squared at 5\% }\end{array}$ \\
\hline Condition (V+H or V) & V+H has significantly higher togetherness & 20.8 \\
\hline Gender & Female higher than male & 25.7 \\
\hline Age & Negative association & 17.8 \\
\hline Computer usage & Positive association & 8.3 \\
\hline
\end{tabular}

As part of the personality profiles, subjects were asked to guess the gender of the remote partner after the experiment for each of the two sensory conditions. Surprisingly, there was a strong difference between the answers for the cases with and without force feedback. The results show that subjects associated the haptic feedback with the 'male' gender. When there was no force feedback, seven out of ten subjects perceived that they were collaborating with a female subject. However, when haptic feedback was added, nine out of ten subjects perceived that they were collaborating with a male subject $(\mathrm{p}<0.01)$. While this result is quite interesting, more detailed investigation needs to be conducted before drawing any reliable conclusions.

\section{Conclusions}

Investigating haptic interactions have applications in the development of effective multi-modal shared virtual worlds, teleoperated systems, and haptic communication protocols on the Internet and in designing intelligent machines for internet-based training. Specifically, the proposed research provides a necessary foundation for the incorporation of 'personal' touch and force feedback devices into networked VR systems and the Internet by investigating the haptic interactions that take place between humans in SVEs.

This paper reports the results of an experimental study conducted to examine the extent to which haptic communication, in addition to the usual visual feedback, influences the task performance and sense of togetherness between remote participants, subject's perception of the remote partner's gender and expert behavior (which includes social anxiety and self-confidence, dexterity, and patience) in a SVE. We neglected the time delays that may take place in two networked computers by eliminating the second computer and connecting two monitors to a single computer. We estimate that the outcomes of the same study could be different due to time delays when both computers of the participants were communicating over the network (see Park and Kenyon, 1999).

We have developed a set of performance and subjective scales to quantify the results of our experiments. The results obtained suggest that haptic feedback affect significantly the task performance and the sense of togetherness. The haptic interaction model used in this study to simulate the underlying physics of interactions (e.g. feeling the forces through pulling or pushing) was simple, but a more sophisticated interaction paradigms can be developed to understand the underlying motor-control, cognitive and perceptual processes that occur during these interactions. Sheridan (1992b) describes several paradigms for remote control of physical systems by humans through computers with applications in teleoperation as well as planning and automation of assembly tasks. One can explore the utility of (a) statistical learning models, (b) game theory, and (c) fuzzy logic techniques to better understand the role of motor-control, cognitive, and perceptual processes that take place between participants during haptic interactions in SVEs. For example, learning models can be used to explain how well the subjects learn to perform a collaborative task that involves touching and manipulating objects in shared environments. Game theory can be useful in modeling haptic negotiations that take place between subjects or between a subject and a computer. Also, we can program the haptic device to make one subject 'strong' (i.e. one participant applies more force to the other when he/she pushes or pulls) and his/her partner 'weak'. Then, a set of rules, based on fuzzy logic, can be implemented to investigate the relations between haptic feedback and gender and emotional experience. For example, the variables of the constructed interaction model can be coded into fuzzy elements that range from strong to weak, expert to beginner, shy to aggressive and the results of the model can be correlated with the gender of the participant. 


\section{Acknowledgements}

Part of this research, including the development of the VE set-up, was supported by a grant from Office of Naval Research. Mel Slater's work in this research was part of his involvement in the European ACTS COVEN project (Collaborative Virtual Environments). He would like to thank Nat Durlach for providing the opportunity for him to spend a short sabbatical period at MIT's Research Laboratory of Electronics.

\section{References}

BARFIELD, W., FURNESS T. 1995. Virtual Environments and Advanced Interface Design, Oxford University Press, NY.

BASDOGAN, C., HO, C., AND SRINIVASAN, M.A. 1997. A Ray-Based Haptic Rendering Technique for Displaying Shape and Texture of 3D Objects in Virtual Environments. ASME Winter Annual Meeting, Dallas, TX, DSC-Vol. 61, 77-84.

BASDOGAN, C., HO, C., SLATER, M., SRINIVASAN M.A. 1998. The Role of Haptic Communication in Shared Virtual Environment, Proceedings of the Third PHANToM Users Group Workshop, Dedham, MA, Oct. 03-06, 1998. (see http://www.ai.mit.edu/conferences/pug98 ).

BENFORD, S., GREENHALG, C., REYNARD, G., BROWN, C., and KOLEVA, B. (1998) Understanding and Constructing Shared Spaces with Mixed Reality Boundaries, ACM Transactions on Computer-Human Interaction, 5, 3, 185-223.

BROLL, W. 1995. Interacting in Distributed Collaborative Virtual Environments, IEEE Proceedings, 148-155.

CHOI, H.R., CHOI, B.H., RYEW, S.M. 1997. Haptic Display in Virtual Collaborative Workspace Shared by Multiple Users, IEEE International Workshop on Robot and Human Communication, 478-483.

DAS, H. et al. 1992. Operator Performance with Alternative Manual Control Modes in Teleoperation, Presence, 1, 2, 201-218.

DURLACH, N., SLATER, M. 2000. Presence in Virtual Environments and Virtual Togetherness, Presence: Teleoperators and Virtual Environments, 9(1).

ELLIS S.R. 1992. Nature and Origin of Virtual Environments: A Bibliographic Essay, Computing Systems in Engineering, 2, 4, 321-347.

HELD, R.M., DURLACH, N.I., 1992. Telepresence, Presence: Teleoperators and Virtual and Real Environments, 1, $1,109-112$.

HO, C., BASDOGAN, C., SLATER, M., DURLACH, N., SRINIVASAN, M.A. 1998. An Experiment on the Influence of Haptic Communication on the Sense of Being Together, The British Telecom Workshop on Presence in Shared Virtual Environments, Ipswich, Suffolk, June 10-11, 1998.

HO, C., BASDOGAN C., SRINIVASAN, M.A. 1999. An Efficient Haptic Rendering Technique for Displaying 3D Polyhedral Objects and Their Surface Details in Virtual Environments, Presence: Teleoperators and Virtual Environments, 8, 5, 477-491,

JASNOCH, U., ANDERSON, B., JOSEPH, H. 1996. Shared 3D Environments within a Virtual Prototyping Environment', IEEE Proceedings of WET ICE'96, 274-279.

LEARY, M. 1983. Social Anxiousness: The construct and its measurement, Journal of Personality Assessment, 47, 66-75. 
MACEDONIA, M., NOLL, S. 1997. A Transatlantic Research and Development Environment, IEEE Computer Graphics and Applications, March-April, 76-82.

NORMAND, V., BABSKI, C., BENFORD, S., BULLOCK, A., CARION, S., FARCET, N., FRECON, E., HARVEY, J., KUIJPERS, N., MAGNENAT-THALMANN, N., RAUPP-MUSSE, S., RODDEN, T., SLATER, M., SMITH, G., STEED, A., THALMANN, D., TROMP, J., USOH, M., VAN LIEMPD, G., AND KLADIAS, N, 1999. The COVEN Project: Exploring Applicative, Technical and Usage Dimensions of Collaborative Virtual Environments, Presence: teleoperators and virtual environments, 8, 2, 218-236.

PARK, K. S., KENYON, R., 1999. Effects of Network Characteristics on Human Performance in a Collaborative Virtual Environment, IEEE Virtual Reality Conference, 104-111.

SALISBURY, J. K., SRINIVASAN, M. A. 1997. Phantom-Based Haptic Interaction with Virtual Objects. IEEE Computer Graphics and Applications, 17, 5.

SHERIDAN, T. 1992a. Musings on Telepresence and Virtual Presence, Presence: Teleoperators and Virtual and Real Environments, 1, 1, 120-125.

SHERIDAN, T. 1992b. Telerobotics, Automation, and Human Supervisory Control, The MIT Press, Cambridge, MA.

SCHROEDER , R. 1997. Networked Worlds: Social Aspects of Multi-User Virtual Reality Technology Sociological Research Online, 2, 4, http://www.socresonline.org.uk/socresonline/2/4/5.html

SLATER, M., A. SADAGIC, M. USOH, R. SCHROEDER (2000) Small Group Behaviour in a Virtual and Real Environment, Presence: Teleoperators and Virtual Environments, 9,1, 37-51.

SRINIVASAN, M.A., BASDOGAN, C. 1997. 'Haptics in virtual environments: taxonomy, research status, and challenges', Computers and Graphics (Special Issue on 'Haptic Displays in Virtual Environments'), 21, 4, 393-404.

STANNEY K.M., SALVENDY, G. 1998a. 'Aftereffects and Sense of Presence in Virtual Environments: Formulation of a Research and Development Agenda', International Journal of Human-Computer Interaction, 10, 2, 135-187.

STANNEY K.M., MOURANT R.R., KENNEDY R.S. 1998b. 'Human Factors Issues in Virtual Environments: A review of the Literature', Presence, 7, 4, 327-351.

STEED, A., M.SLATER, A.SADAGIC, J.TROMP and A.BULLOCK, 1999. Leadership and Collaboration in Virtual Environments, IEEE Virtual Reality, Houston, March 1999.

TROMP, J., STEED, A., FRECON, E. BULlOCK, A., SADAGIC, A., SLATER, M., 1998. Small Group Behaviour in the COVEN Project, IEEE Computer Graphics and Applications, 18, 6, 53-63.

WATERS, R., BARRUS, J. 1997. ' The Rise of Shared Virtual Environments', IEEE Spectrum, March, 20-25.

WELCH, R.B., BLACKMON, T.T., LIU, A., MELLERS, B.A., STARK, L.W. 1996. 'The Effects of Pictorial Realism, Delay of Visual Feedback, and Observer Interactively on the Subjective Sense of Presence', Presence: Teleoperators and Virtual and Real Environments, 5, 3, 263-273. 